\title{
Conceptual blending as a tool for analyzing group discourse
}

\author{
Jessica R. Hoehn, ${ }^{1}$ Noah D. Finkelstein, ${ }^{1}$ and Ayush Gupta ${ }^{2}$ \\ ${ }^{1}$ Department of Physics, University of Colorado, 390 UCB, Boulder, CO 80309 \\ ${ }^{2}$ Department of Physics, University of Maryland, 4150 Campus Dr, College Park, MD 20742
}

\begin{abstract}
We demonstrate that Fauconnier and Turner's theory of conceptual blending, originally a theory of the mind that focuses on the individual, can be used as a tool for analysis of group discourse processes. Building on sociocultural theories that consider cognition a social process, we propose blending theory as a descriptive and analytic tool for use in examining the processes of collective reasoning. We present data from focus groups at University of Colorado Boulder in which Modern Physics students engage in reasoning about quantum phenomena and negotiate ontological conceptions of quantum entities such as electrons and photons (i.e. negotiating what kind of a thing an electron or photon is). We present a conceptual blending analysis of group discourse within this context, and show how conceptual blending theory can be used to describe both collectively constructed blends and more traditional individual blends.
\end{abstract}

\section{INTRODUCTION}

Physics education researchers have spent a long time studying student sense-making and understanding both to develop theories of mind and to improve curricula. While attention has often focused on the individual student, some work attends specifically to sense-making in groups [1-3]. Studying student reasoning in a group context is important and useful to the field, as physics curricula are often designed around collective sense-making, and collaborative work is often a goal of our courses. As one part of a larger study on student reasoning in quantum mechanics (QM), we attend to how the ontology of quantum entities - their characterization as having properties of matter and/or waves-is constructed within group discourse. We do this with both theoretical goals (understanding collective reasoning in itself) and curricular implications in mind. In order to explore the dynamics of collective negotiation about the ontologies of quantum entities, we utilize conceptual blending [4] as an analytic tool. Although the original theory of conceptual blending is a theory of cognition that ascribes blends to individual minds, we demonstrate how this framework can be applied to group settings. In doing so, we model group conversation with collectively constructed blends and consider mental spaces as ideas that are socially distributed. The purpose of this paper is to make a methodological contribution to physics education research by providing a "proof of principle" for using blending as a tool for analyzing group discourse.

\section{CONCEPTUAL BLENDING}

Conceptual blending is a theory of cognition developed by Fauconnier and Turner (F\&T) [4] that describes the dynamic process by which mental spaces (conceptual packets constructed in-the-moment) combine to form a blend space. Two (or more) input spaces have elements which are projected to the blend space, and new meaning emerges when the blend is elaborated, a process described as a mental simu- lation or "running of the blend". For F\&T, conceptual blending is an unconscious cognitive activity present in every aspect of human life, and mental spaces can be used to "model dynamic mappings in thought and language" [4]. They also emphasize that the standard blend diagrams are static representations of this dynamic and imaginative process. Within PER, the conceptual blending framework has been utilized in several ways [5-9], including investigating students' understanding of wave propagation [5] and modeling coordinated use of ontological metaphors for energy [6]. In this paper we will demonstrate how this framework can be a viable tool beyond the analysis of an individual's cognition to describe collective reasoning as we explore the dynamics of group discourse about ontological conceptions of photons.

\section{DATA COLLECTION AND ANALYTIC APPROACH}

Our data come from a transformed Modern Physics course at University of Colorado Boulder, which serves as an introduction to QM for engineering students. The curriculum focuses on the conceptual foundations and real world applications of QM [10], and explicitly addresses physical interpretation of quantum phenomena [11]. One of the explicit course goals is for students to engage in scientific discourse with their peers around conceptual and interpretive themes. We ran biweekly focus groups of students enrolled in this course to study in-the-moment student reasoning and the nature of students' ontologies. One group of three students and one group of four students participated in six hour-long focus group sessions over the course of the semester. The students were presented with prompts directly designed to investigate student reasoning on topics drawn from the class. The students were encouraged to think aloud and discuss with their peers, and two researchers were present to facilitate the discussion. These sessions were video recorded. Preliminary analysis included identifying specific episodes where students seemed to be negotiating the ontology of quantum entities. We transcribed the tagged episodes and coded segments of collabora- 
tive discourse from a conceptual blending perspective to explore how blending theory can help us describe the collective reasoning in terms of blended spaces and blending processes.

We model a conversation between three students as a collective blend. In this focal episode, which comes from the first ten minutes of the third focus group session, three students, pseudonymed Eric (white male), Tara (female; chose not to identify race/ethnicity), and Bryan (white male), are negotiating their ontological conceptions of a photon. The students were given a schematic of a classic Mach-Zehnder interferometer with a single photon source (a simple interferometer set up with one beam splitter, two mirrors, and two detectors) along with the following questions: 1) What is going on when photons are sent through the experiment? 2) How would this experiment be different with classical particles or EM waves? 3) How do you think about the energy of the photon in this situation? The students had access to the "Interferometer experiments with photons, particles and waves" simulation from University of St. Andrews [12] and were encouraged to utilize it to help them answer the questions. We showcase this particular episode that covers a widely applicable topic (photons) and is representative of the types of discussions analyzed. We present our analysis of this episode to support the claim that a collective conceptual blending framework is a useful analytic tool for capturing: 1) collective construction of meaning and 2) dynamics of group discourse.

\section{ANALYSIS OF FOCAL EPISODE}

\section{A. Collective Construction of Meaning}

At the start of the focal episode, Eric suggests that the beam splitter splits the photon. Tara refines Eric's description, contesting the notion that the photon can split, and Eric subsequently responds to the correction:

Eric: Umm...well when photons go through the beam splitter, and it splits the photon, and then, when it hits the detectors it goes into one of them randomly.

Bryan: Yeah, it's a 50-50 shot, which one it's detected by.

Tara: But, I-I think you have to be careful about your language there, because the photon itself I don't think is split, it has an equal probability of going through either of these paths, but the photon is still like... a photon [cupping hands in gesture of a contained substance]

Eric: So it's not physically split, but like, it's in the ambiguous state of being-

Tara: -It's superimposed-

Eric: -on both paths [nods]

Thus far in the conversation, the group's conception of a photon (as evidenced by their speech and collective utterances) is a contained entity that hits one of the two detectors (with equal probability) and while it does not physically split, it is "superimposed", a term that will come to have a slightly different meaning for each of the students as they continue to collectively negotiate their ontological conceptions. This characteristic of being "superimposed" (later referred to as being in a superposition state) emerges in the discussion to coordinate the tension between the photon not splitting but having equal probability of hitting either detector. Next in the conversation, Eric and Tara jointly construct the understanding that the photon is "superimposed" until it chooses one detector. Tara says of the photon, "it decides one of these [paths] based on probability" and Eric reiterates by saying that "it just chooses one [path] and goes entirely into that one". In this statement, Eric seems to have taken up Tara's idea that the photon does not actually split at the beam splitter.

The students then attend to the second prompt asking them to articulate how the photon is different from a classical particle or wave. Referring to a classical particle, Tara begins the discussion (particle characteristics are underlined with a straight line and wave characteristics with a wavy line):

Tara: Like, if-if you think of this as like a, like a literal ball, it can either go like that, or like that [drawing on paper]. And so can the photon, but not as physically as this would. Like this would exist at this path the whole time-

Eric: Yeah, you would be able to like see which path it was on as it traveled.

Tara: Right, but the photon's like, I'm gonna do one of these things...we'll find out.

Eric: [Laughs] Um, versus with EM waves, it would actually go down both paths.

Bryan: Yeah, it's actually split.

Eric: So like, it's the two extremes, like with the particle it would go down one path only and with the wave it would go down both paths, and with like the photon it neither-it does like something in between where it just super-positions itself along both paths and then goes into one of them.

Tara: Right, like with the EM wave you have half the wave going on one part and half the way-wave on the other

Here we see students engaging in metacognitive reasoning to explicitly identify the classical particle and electromagnetic wave characteristics that form two extremes between which the photon characteristics lie. They continue by identifying how the photon differs from these entities:

Eric: Yeah, so we know that it doesn't act like a wave because it doesn't get detected by both detectors.

Tara: Right.

Eric: Is there any reason we know it doesn't act like a particle?

Tara: Because we don't know where it is. It could be on either of those paths...So if you look at it [doing something on the computer]...particle...it's very clearly going one way or another. But with the photon, it's superimposed on both of these paths.

To model how the students collectively organize the properties of the photon in this context as drawing on both particle and wave characteristics, we map the group's discussion onto 


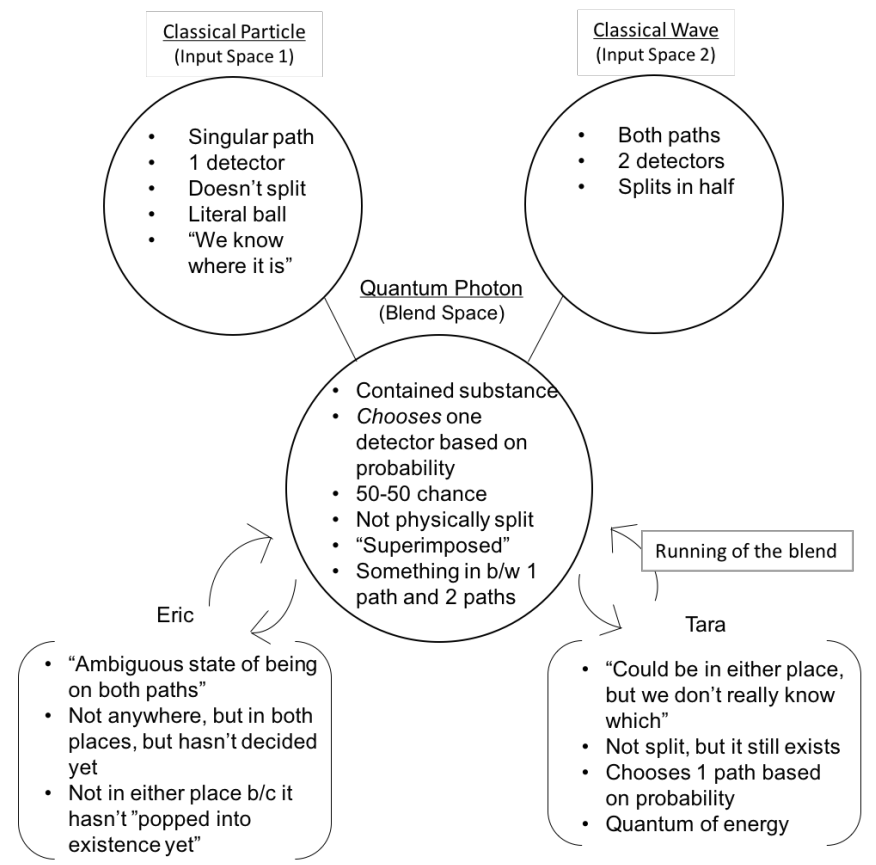

FIG. 1. We model the group discourse with a collectively constructed blend of classical particle and classical wave input spaces. The elements of the classical particle, classical wave, and quantum photon spaces are defined collectively by the students. Two of the three students (Eric and Tara) run the shared blend and arrive at different interpretations of "superposition".

the blend diagram in Figure 1. Classical particle and classical wave form the input spaces-with elements defined by the students' collective reasoning and agreement in lines 1645-that merge to form the photon blend. The group's conception of the photon begins to emerge at the outset of the conversation when they define that a photon is not physically split (line 11), it goes into one of the two detectors with equal probability (lines 3-5), and that it is "superimposed" (lines 13, 45). Through negotiation of the superposition element, the students refine their ideas about how the photon behaves. That is, to coordinate between the photon not being able to physically split at the beam splitter and the fact that it has equal probability of hitting each detector, the students agree that the photon chooses one detector based on probability.

Unlike traditional conceptual blending analyses, here each of the three students has contributed to the construction of the blend spaces as well as defined the input elements. We map the collective ontological negotiation onto the blend diagram in Figure 1, noting that our use of the term "mental space" is different from that originally defined by F\&T. In our context, the quantum photon blend space is not something held in one individual's mind, but rather is a socially distributed conception constructed and utilized by the group to make sense of the quantum phenomenon in question. The blend space ontology of the photon emerges first in the discussion, then the students articulate the input space elements, effectively con- structing an ontology of the photon that draws on both particle and wave categories but does not fully map onto either.

\section{B. Dynamics of Group Discourse}

The two input spaces and blend space in Figure 1 represent the shared meaning constructed by the group-each of the three students contributed elements to these mental spaces and agreed on the ontologies thus far defined. As the conversation continues, new meaning arises as the students try to make sense of the quantum photon blend space:

Eric: Yeah, it's weird to think about where the photon is when it's in this like superposition state of not being anywhere. But also being in both places, but not really, because it hasn't decided yet.

Bryan: Yeah.

Eric: It's like it goes back and changes history when it hits. But, I don't think that's a good way to think about it. [Laughs] I don't think that's accurate.

Tara: Well, I mean, I always think of the energy of the photon always being [gestures contained substance with her hands], like together. Like it can't really be split 'cause it's a quantum of energy.

As Eric attempts to make sense of the behavior of the photon, he brings in a new idea that the photon changes history when it hits a detector (line 51). He then immediately distances from this idea with laughter giving some sense that a photon behaving this way would be "too weird"; Eric is still negotiating his understanding as he runs the already accepted blend. We also see new meaning emerging for Tara as she evokes the idea of quantization of energy (line 57) in order to elaborate on the element of "not physically split". Through the further elaboration of the collectively constructed quantum photon blend, Eric and Tara individually bring in new ideas to make sense of the superposition aspect of the photon's behavior. In doing so, they draw different interpretations of what it means for the photon to be in a state of superposition (represented as the brackets in Fig. 1). We cannot discern how Bryan is thinking about the superposition element because he takes much fewer turns of talk. At the end of the ten-minute clip, Eric and Tara each articulate where they stand in regards to the meaning of superposition:

Eric: ...because we don't really know the position of the photon, I don't really know where the energy is. I assume it's wherever the photon is...So I guess the energy is also in a sup-state of superposition.

Tara: I think that's a good way of thinking about it. Like it could be in either place, all of it could be in either place, but we don't really know which, and it's not split, but it still exists.

Eric: And it's not actually in either of those places yet, because it hasn't been detected yet, so it doesn't-it hasn't like popped into existence yet. If that's the correct way of thinking about that...

Again Eric brings in new ideas (lines 59,68) that may or may 
not be rejected in the process of sense-making about the quantum photon. In running the blend, Eric elaborates on his original statement of the "ambiguous state of being on both paths" (lines 12-14) by talking about the photon as "not being anywhere", but also "being in both places", while having "not decided yet" (lines 47-49). This contrasts Tara's descriptions that the photon is a "quantum of energy" (line 57) that could be in either place but "we don't really know which" (line 64). In mapping the group's conversation onto the blending framework, we can explore the collective construction of shared meaning and the dynamic processes through which individual students elaborate in different ways.

\section{DISCUSSION}

In this novel application of blending, we model the collective construction of blends; All three students contribute to defining the input and blend spaces. The students have shared meaning around the elements of classical particle, classical wave, and quantum photon evidenced by the agreement in the conversation (e.g. "Right", "Yeah"), the repetition of ideas, and the coherence of the conversation (building off of each other's ideas). We acknowledge that while the episode was dominated by a group of two students (Eric and Tara), the third student (Bryan) does contribute to the collective understanding constructed in the group. We make no claims about the bounds on group size (other than $\mathrm{N}>1$ ) for this collective blending analysis, and note that it would likely depend on the context in which the group were operating.

Mapping the students' conversation onto the conceptual blending framework helps to elucidate the dynamic and nuanced processes within the group's meaning making, advancing our understanding of how students negotiate the ontological meaning of constructs in physics [13]. Although not evident in the diagram (a static representation of a dynamic process), the idea that the photon "splits" is rejected from the blend multiple times. Eric continually brings up the idea of splitting and each time it is ultimately rejected or re-framed within the group. For example, in the first few moments of the conversation, Eric's idea that the beam splitter "splits the photon" is rejected from the blend space. This dynamic process happens through Tara's correction of Eric's language (lines 6-7), and ultimately results in the blend space element "not physically split". Each time this "splitting" is questioned and rejected by the group, new meaning emerges for the students.

The quantum photon blend space arises first in the conversation, and the "superimposed" element emerges as a way for the group to coordinate between the properties that the photon is not physically split, but that it has equal probability of going into either detector. The students first agree on their conception of the photon (quantum photon blend space) and then collectively negotiate the meaning of said photon.

Modeling the conversation through a conceptual blending framework and identifying the associated running of the blend processes enables us to explore where the students have shared meaning and how the elaboration of that shared meaning results in different interpretations for two of the three students. In looking at the conversation holistically as well as attending to specific utterances by the students, it is clear that Eric and Tara come away from the discussion with different interpretations of what it means for the photon to be in a state of superposition. Tara maintains a classical ignorance interpretation - the photon takes one path or the other, and we simply do not know which until it is detected. Eric on the other hand is less certain in his ontological stance, stating that the photon isn't anywhere because it doesn't exist yet, but that it also could be on both paths. At the end of this episode, Eric is continuing to turn the gears of elaboration and negotiate the behavior of the photon for himself through conversations around shared and unshared meaning in the group.

As we seek to foster collective student reasoning around curricula, we can use conceptual blending theory as a tool for capturing and understanding student reasoning in these shared environments. In future work we will explore how refined models of student understanding using conceptual blending can help in the design of curriculum.

\section{ACKNOWLEDGMENTS}

The authors thank Andrew Elby, Benjamin W. Dreyfus, Erin Ronayne Sohr, and the CU PER group. Work supported by NSF TUES \#1322734, NSF \# 1323129 and an NSF GRF.
[1] R.E. Scherr and D. Hammer, Cog. Instr. 27, 2 (2009).

[2] B.W. Frank and R.E. Scherr, Phys. Rev. Phys. Educ. Res.8, 2 (2012).

[3] B. Barron, J. Learn. Sci. 9, 4 (2000).

[4] G. Fauconnier and M. Turner, Basic Books, New York (2002).

[5] M.C. Wittman, R.N. Steinberg, and E.F. Redish, Phys. Teach. 37, 1 (1999).

[6] B.W. Dreyfus, A. Gupta, and E.F. Redish, Int. J. Sci. Educ. 37, 5-6 (2015).

[7] N.S. Podolefsky and N.D. Finkelstein, Phys. Rev. Phys. Educ. Res. 3, 1 (2007).

[8] D. Hu and N.S. Rebello, Phys. Rev. Phys. Educ. Res. 9, 2
(2013).

[9] E. Gire and E. Price, Phys. Rev. Phys. Educ. Res. 10, 2 (2014).

[10] S.B. McKagan, K.K. Perkins, and C.E. Wieman, PERC Proc., AIP Press (2006).

[11] C. Baily and N.D. Finkelstein, Phys. Rev. Phys. Educ. Res. 11, 2 (2015).

[12] http://www.st-andrews.ac.uk/physics/quvis/simulationstwolev/IOP, (Retrieved March 2015).

[13] D.T. Brookes and E. Etkina, Phys. Rev. Phys. Educ. Res. 3, 1 (2007). 